\title{
Autoregressive Integrated Moving Average (ARIMA) Modeling of Time Series of Local Telephone Triage Data for Syndromic Surveillance
}

\author{
Micael Widerström*1,2, Maria Omberg ${ }^{1}$, Martin Ferm ${ }^{3}$, Ann-Katrine Pettersson ${ }^{4}$, Malin \\ Rundvik Eriksson ${ }^{1}$, Ingela Eckerdal ${ }^{4}$ and Johan Wiström ${ }^{5,6}$ \\ 'Department of Communicable Disease Control and Prevention, Jämtland County Council, Östersund, Sweden; ${ }^{2}$ Department \\ of Clinical Microbiology, Unit of Clinical Research Center- Östersund, Umeå University, Umeå, Sweden; ${ }^{3}$ Centre of Registers in \\ Northern Sweden, Umeå University, Umeå, Sweden; ${ }^{4}$ Swedish Health Care Direct 1177, Jämtland County Council, Östersund, \\ Sweden; ${ }^{5}$ Department of Clinical Microbiology, Infectious Diseases, Umeå University, Umeå, Sweden; ${ }^{6}$ Department of \\ Communicable Disease Control and Prevention, Västerbotten County Council, Umeå, Sweden
}

\section{Objective}

Our aim was to use telephone triage data to develop a model for community-level syndromic surveillance that can detect local outbreaks of acute gastroenteritis (AGE) and influenza-like illness (ILI) and allow targeted local disease control information.

\section{Introduction}

National telephone health advice service data have been investigated as a source for syndromic surveillance of influenza-like illness and gastroenteritis (1-3). Providing a high level of coverage, the system might serve as an early outbreak detection tool. We have previously found that telephone triage service data of acute gastroenteritis was superior to web queries as well as over-the-counter pharmacy sales of anti-diarrhea medication to detect large water- and foodborne outbreaks of gastrointestinal illness in Sweden during the years 20072011 (4). However, information is limited regarding the usefulness, characteristics, and signal properties of local telephone triage data for monitoring and identifying outbreaks at the community level.

\section{Methods}

Weekly aggregated local telephone triage data (Swedish Health Care Direct 1177) collected from November 2007 to December 2012 in eight municipalities in Jämtland County, Sweden, were analyzed by seasonal autoregressive integrated moving average (ARIMA) modeling of time series of the two common syndromes AGE ("diarrhea" or "nausea or vomiting") and ILL ("fever" and "cough"). Data analysis was done using the free software R (a language and environment for statistical computing) and its forecast package version 3.0.2013-0512 (http://www.r-project.org). Selection of model parameters was assessed using Akaike's Information Criterion (AIC) and the model performance was evaluated using mean squared error (MSE). Upper limits for prediction intervals were set at $90 \%$ and $95 \%$, respectively, and were used to generate signals. Upper limits for prediction intervals were set at $90 \%$ and $95 \%$, respectively, and were used to generate signals.

\section{Results}

The seasonal ARIMA model showed good performance in predicting future values of AGE and ILI and was able to detected significantly elevated values for AGE and ILI in 7 of 8 communities during the 2012-2013 influenza and calicivirus season.

\section{Conclusions}

Our seasonal ARIMA model using local telephone triage data detected AGE and ILI outbreak signals in the majority of the investigated communities. This forecast model can prove to be an important tool for monitoring local levels of AGE and ILI to enable early detection of outbreaks. This information may also aid implementation of preventive measures by healthcare professionals and the public.

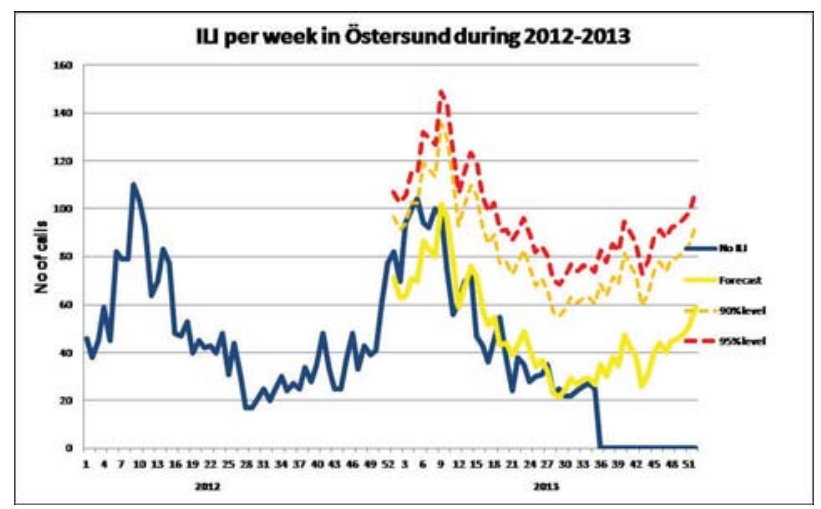

\section{Keywords}

Syndromic surveillance; Outbreak detection; Data analysis; Disease outbreak; Public Health Surveillance

\section{Acknowledgments}

This work was funded by the Swedish Civil Contingencies Agency (MSB).

\section{References}

1. Doroshenko A, Cooper D, Smith G, Gerard E, Chinemana F, Verlander $\mathrm{N}$, et al. Evaluation of syndromic surveillance based on National Health Service Direct derived data-England and Wales. MMWR Morb Mortal Wkly Rep. 2005 Aug 26;54 Suppl:117-22.

2. Smith S, Elliot AJ, Mallaghan C, Modha D, Hippisley-Cox J, Large $\mathrm{S}$, et al. Value of syndromic surveillance in monitoring a focal waterborne outbreak due to an unusual Cryptosporidium genotype in Northamptonshire, United Kingdom, June - July 2008. Euro Surveill.15(33):19643.

3. Yih WK, Teates KS, Abrams A, Kleinman K, Kulldorff M, Pinner R, et al. Telephone triage service data for detection of influenza-like illness. PLoS One. 2009;4(4):e5260.

4. Andersson T, Bjelkmar P, Hulth A, Lindh J, Stenmark S, Widerstrom M. Syndromic surveillance for local outbreak detection and awareness: evaluating outbreak signals of acute gastroenteritis in telephone triage, web-based queries and over-the-counter pharmacy sales. Epidemiol Infect. 2013 May 15:1-11.

\footnotetext{
*Micael Widerström

E-mail: micael.widerstrom@jll.se
} 